MS32-O5 In the jungle, the mighty jungle, the crystals grow tonight!

Ulrike Werthmann ${ }^{1}$

1. Boehringer Ingelheim GmbH, Germany

email: ulrike.werthmann@boehringer-ingelheim.com

The process of crystal formation can sometimes be described as a walk through a dense jungle with no clear trail or map to guide you. Unexpected effects and unanticipated formation of "fancy crystals" are observed and this makes the life as scientist within a pharmaceutical industry very exciting! To get orientated in this "jungle" it is necessary to explore a broad experimental space to achieve the goal of identifying the form of an active pharmaceutical ingredient (API) with the most favorable properties for drug development. Using a High Throughput Crystallization Approach ( $\mu \mathrm{L}$-scale) the crystallization conditions can be defined. A large number of different crystallization conditions may be tested and up to 384 experiments can be performed within one run using 96-well plate formats (4 plates). Different solvents and solvent mixtures in combination with a variety of counter ions (for ionisable drugs) and/or cocrystal formers lead to a quick overview of the crystallization behavior of the API. This screening tool may be utilized for polymorph screening as well as for salt- and co-crystal-screening. All solid forms resulting out of this HT-screen (approx. 3mg/well) need to be analyzed by e.g. X-ray powder diffraction to check whether the compound crystallized or not. Furthermore, in order to characterize the resulting crystalline forms and to draw conclusions from the large amount of data (more than 100 patterns from a single experiment), a specific software tool is needed to handle and maintain an overview of the resulting "data jungle". A case study will be presented to get a bit better orientation in the mighty jungle of crystal formation.

Keywords: API, HT-crystallization, Pharmaceutical Industry
MS33 Hot structures of small

molecules

Chairs: Marijana Đaković, Andreas Roodt

\section{MS33-01 How to fill MOFs with different flavours}

Alessia Bacchi ${ }^{1}$, Paolo Pelagatti ${ }^{1}$, Davide Balestri ${ }^{1}$, Stefano Canossa ${ }^{1}$, Patrick Scilabra

\section{Dipartimento di Chimica, Università di Parma, Italy}

email: alessia.bacchi@unipr.it

The scope of this work is to find a systematic way to embed small molecular aggregates inside porous crystalline materials, with the multiple aims to explore the structural aspects of nanoconfinement, and of the stabilization of guest molecules inside the cavities of the structure. The feasibility of this approach stems from both the recent report that describes the structural determination of single molecules trapped inside a microporous framework [1] and the various reports showing the inclusion of species such as metal nanoparticles or polyoxometalates into mesoporous MOFs [2]. MOFs are highly versatile materials that are made by connecting metal ions with prefixed coordination geometry with rigid ligands acting as spacers, hence affording three-dimensional coordination polymers. The accurate design of the building units allows to design porous MOFs, obtaining cavities of considerable size, which usually accommodate loosely bound solvent molecules. The guest that we are considering here are some important compounds for the human health and nutrition which occur as liquids at room temperature, some organometallic precursors of inorganic oxides, and organometallic compounds potentially active in catalysis. We initially focused on a collection of already known MOFs, and we determined the interaction mode of some of the guests inside the pores (Figure 1), and the results will be shown. We then moved to the design of new organic linkers in order to better tune the topology and functionality of the MOF network. In particular, we aimed to decorate the inner cavities with hydrogen bond and halogen bond active functional groups, which could serve as anchoring points for the guests. A small library of linkers was synthesized: one group is composed by flexible aminocarboxylic linkers, the second one is characterized by rigid amidic bonds and pyridine as coordinative function. These ligands were used for the synthesis of novel MOFs; due to the nature of the ligands these frameworks resulted quite flexible. Their structure and inclusion properties will be illustrated.

[1] Y. Inokuma et al., Nature, 2013, 495, 461-466 [2] C. Rösler, R. A. Fischer, CrystEngComm, 2015, 17, 199-217 [3] H.C. Zhou et al., J. Am. Chem. Soc,.2007, $129,1858-1859$ 


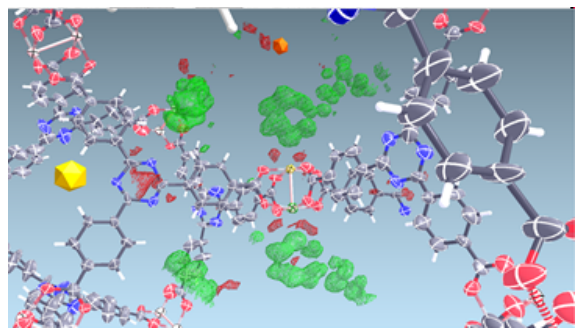

Figure 1. Experimental electron density of nicotine included inside the MOF known as PCN6 [3].

Keywords: crystal engineering, MOF, inclusion compounds

\section{MS33-O2 Solid-state transition-metal photoactive materials - tracing structure-property relationships via combined spectroscopic and crystallographic approaches}

Katarzyna N. Jarzembska ${ }^{1}$, Radosław Kamiński ${ }^{1}$

1. Biological and Chemical Research Centre, Department of Chemistry, University of Warsaw, Żwirki i Wigury 101, 02-089 Warsaw, Poland

email: katarzyna.jarzembska@gmail.com

Coordination complexes can be described as 'hot' small molecules due to their rich electronic and luminescent properties. $^{1}$ Especially interesting here are $\mathrm{d}^{8}$ or $\mathrm{d}^{10}$ transition-metal systems, which frequently constitute the active sites of both biological and chemical catalysts, have versatile applications in solar-energy conversion and other fields ranging from molecular electronics and photocatalysts to light-emitting diodes (LEDs) and biolabels. It is, thus, of high importance to sensibly control optical properties of such materials at the molecular level, in order to apply this knowledge to generate materials with particular properties across all the size scales from molecules to bulk materials, relevant for specific applications.

It occurs that short metal-metal contacts usually determine the nature of the lowest lying emissive states, and so are crucial to understand physical properties of the respective materials. In this contribution we shall present our most recent results regarding the analysis of charge transfer processes and structure-property relationships of selected coinage metal complexes $(\mathrm{Cu}, \mathrm{Ag}, \mathrm{Pt}, \mathrm{Ni}$, $\mathrm{Fe}$, etc.) in the solid state. ${ }^{2-5}$ For the purpose of our investigations we combined advanced spectroscopic and crystallographic techniques supported by theoretical calculations.

(1) Yam, V. W.-W.; Lo, K. K.-W. Chem. Soc. Rev. 1999, 28, 323. (2) Jarzembska, K. N.; Kamiński, R.; et al. Inorg. Chem. 2014, 53, 10594; Koshevoy, I. O.; Shakirova, et al. Dalton Trans. 2011, 40, 7927. (3) Filatov, A. S.; Hietsoi, O.; Sevryugina, Y.; Gerasimchuk, N. N.; Petrukhina, M. A. Inorg. Chem. 2010, 49, 1626. (4) Trzop, E.; Fournier, B.; Jarzembska, K.; Sokolow, J.; Kaminski, R.; et al. Acta Cryst. 2014, A70, C776. (5) Jarzembska, K. N.; Chen, Y.; et al. Phys. Chem. Chem. Phys. 2014, 16, 15792.

Keywords: coordination complexes, charge transfer, photocrystallography 